EPJ Web of Conferences 82, 01015 (2015)

DOI: $10.1051 /$ epjconf/20158201015

(C) Owned by the authors, published by EDP Sciences, 2015

\title{
About the temperature of the thing under the influence of heat radiation of high intensity
}

\author{
D.K. Baktybaeva a and V.E. Yukhnov, s.T.S. \\ Tomsk Polytechnic University, s. Tomsk, Russia
}

The study of the nature of radiant heat, refers to one of the young branches of physics. Broad scientific interest in the study of the invisible heat rays associated with the beginning of transformation in the metallurgical and chemical production of Europe - is in industrialization. The use of steam machines and the associated interest to thermal phenomena influenced in development of the doctrine of the heat. For the first time the concept of thermal radiation is referred to Swedish chemist Carl C. Scheele in his Chemical treatise on air and fire (1777 y.o.) based on many observations. In 1779 y.o. in proceedings of the Pyrometry, German mathematician and physicist Johann Lambert, were described experiments, consistent with the observations Scheele and for the first time experimentally proved that heat rays propagate rectilinearly, and their intensity decreases inversely to the square of the distance. In 1790 y.o. Mark O. Pictet continued the experiment through experiments which were of great importance to determine the nature of radiant heat and the further development of her theory.

It is now known that the thermal radiation is electromagnetic radiation emitted by the substance and emerging due to the energy of thermal motion of atoms and molecules, is one of the modes of transmission of heat energy, along with such species as the thermal conductivity and convection.

Recently, the importance of the study of thermal radiation was connected with natural and technogenic processes, for example, fire risk assessment and the prevention of such emergencies [1, 2]. Open fire is a source of highly intensity thermal radiation. When it is flow of heat radiation significantly affects the rate of spread of fire. As a rule, in view of substantial economic costs, such studies are carried out on numerical models. Therefore, to obtain reliable experimental data in the study of heat sources, open flame of the surrounding objects is an important practical task. The aim of the present work is an experimental studying of an influence of distance from the source with the heat radiance density $130 \cdot 10^{3} \mathrm{BT}^{2}$ on studying sample.

The objectives of the study are to measure the temperature of the sample from different distances of the source of thermal radiation, as well as the definition of the analytical dependence of the temperature of the sample at different distances from the source of thermal radiation.

A scheme of the experimental setup area on which the research was conducted in accordance with figure

1. The installation consists of a source of thermal radiation $\left(T_{0}\right)$, sample temperature sensor $\left(T_{2}\right)$, two sliding screens, and measuring the distance from the source of thermal radiation to the sample with temperature sensor.

\footnotetext{
${ }^{a}$ Corresponding author: dana_-8888@mail.ru
}

This is an Open Access article distributed under the terms of the Creative Commons Attribution License 4.0, which permits unrestricted use, distribution, and reproduction in any medium, provided the original work is properly cited. 


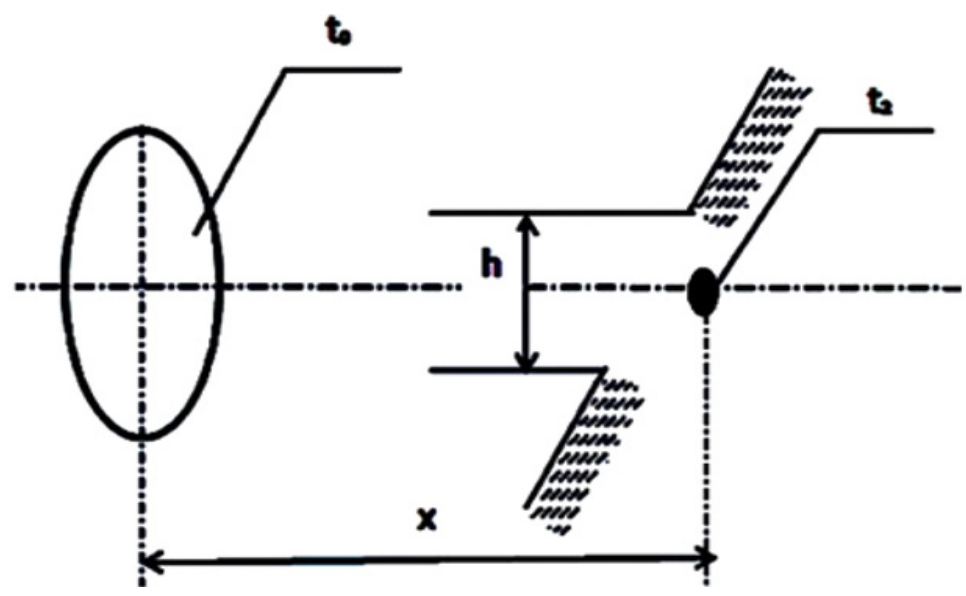

Figure 1. The measuring scheme of the experimental setup: $T_{2}$ - the sample temperature, $\mathrm{x}-$ the distance from the radiation source to the sample, $\mathrm{h}$ - the distance between the screens, $\mathrm{T}_{0}$ - the temperature of the radiation source.

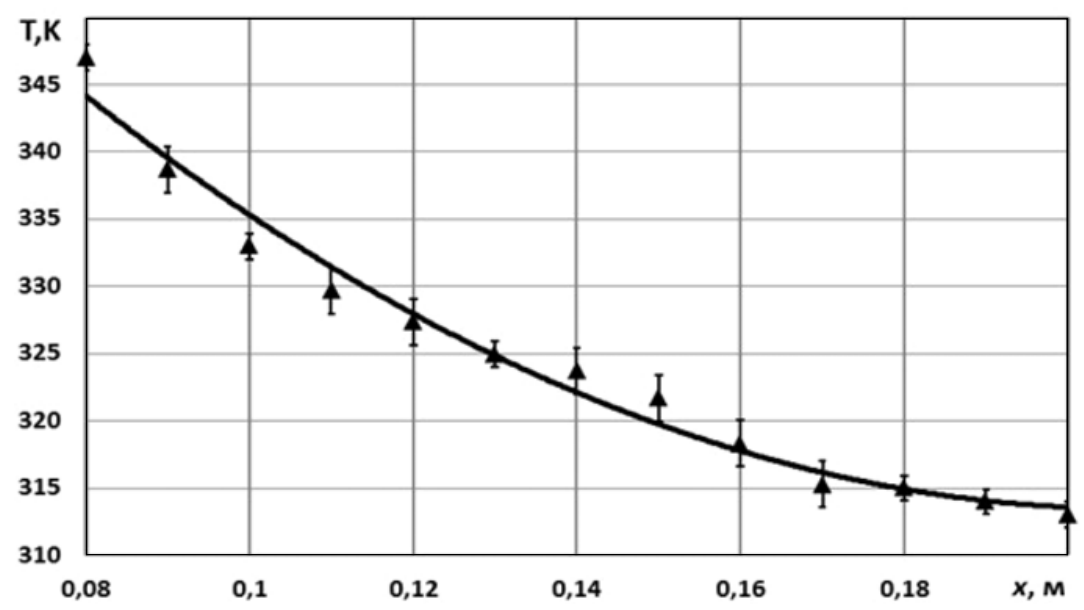

Figure 2. Experimental data of the temperature of the sample $T_{2}$ at different distances $x$ from the radiation source when $h=0,5 \cdot 10^{-2} \mathrm{~m}$.

A source of thermal radiation was the flame of the burner, placed in the flame of a thermoelectric transducer for temperature measurement. The distance $\mathrm{x}$ between the source of thermal radiation and the irradiated sample varies from $8 \cdot 10^{-2} \mathrm{~m}$. to $20 \cdot 10^{-2} \mathrm{~m}$. rates. The Measurement of the steady-state temperature of the sample $\mathrm{T}_{2}$ was conducted at an average temperature of the source of thermal radiation is equal to $=1233,15 \mathrm{~K}$.

Part of the flow of thermal radiation incident on the sample was cut two screens with a gap width $\mathrm{h}=0,5 \cdot 10^{-2} \mathrm{~m}$ Each measurement was performed three times. The results of statistical data processing with regard to the detection and elimination of gross errors (blunders) [3, 4] obtained in accordance with Fig. 2.

In accordance with Fig. 2, the results showed that with increasing distance $\mathrm{x}$ between the source of thermal radiation and the sample, the temperature decreases. The experimental data of the temperature of the sample, located at different distances from the source of thermal radiation, in accordance with 


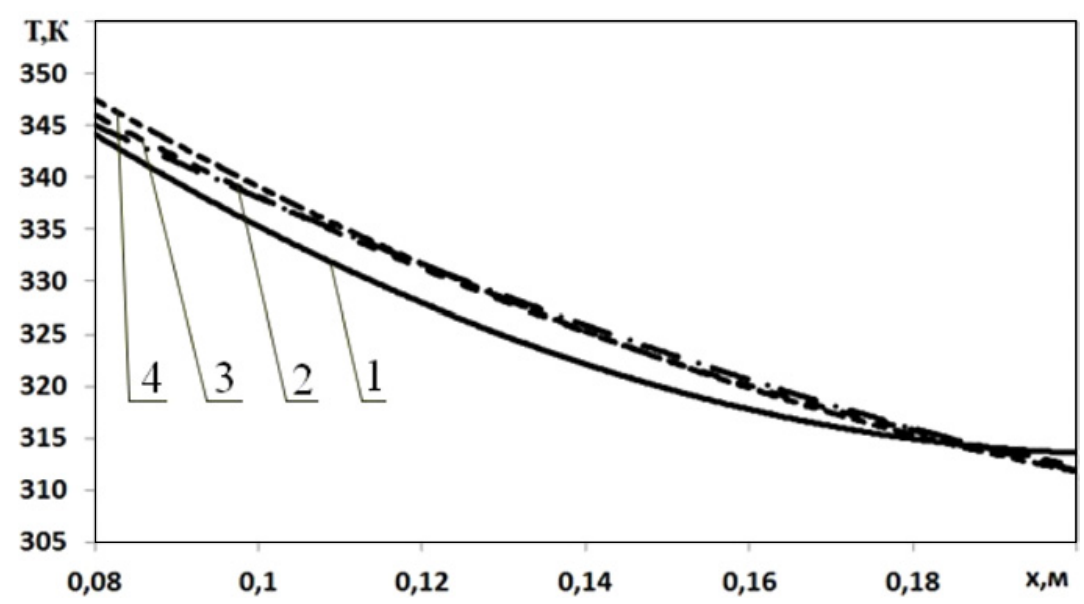

Figure 3. Graphs of temperature change of the sample $\mathrm{T}_{2}$ the distance $x$ when $\mathrm{h}: 1-0,5 \cdot 10^{-2} \mathrm{~m},-2 \cdot 10^{-2} \mathrm{~m}$, $3-4 \cdot 10^{-2} \mathrm{~m}, 4-8 \cdot 10^{-2} \mathrm{~m}$.

Fig. 2, satisfactorily describes the approximate dependence represents a power function

$$
T_{2}(x)=a x^{b}, \mathrm{~K},
$$

where the coefficients $a=2,7461 ; b=-0,648$. The approximation error does not exceed $0,5 \%$ rate.

To clarify the influence of the width of the gap between the screens on the temperature of the irradiated sample held four experience when changing from $0,5 \mathrm{~h} \cdot 10^{-2} \mathrm{~m}$. to $8 \cdot 10^{-2} \mathrm{~m}$. On the basis of experimental data obtained four approximate dependency graphs are shown in Fig. 3, having a form of power function (1). The analysis of the dependence of the temperature sensor $T_{2}$ is the distance between the screens $h$ in the whole range of $x$ positions of the sensor from the radiation source showed that the increase in the width of the gap between the screens $\mathrm{h} 2 \cdot 10^{-2} \mathrm{~m}$. to $8 \cdot 10^{-2} \mathrm{~m}$. leads to an increase in temperature of the sample is not more than $6 \%$ compared with the sample temperature $\mathrm{T}_{2}$ when the width of the gap between the screens $\mathrm{h}=0,5 \cdot 10^{-2} \mathrm{~m}$. Therefore, the temperature sensor is only weakly depend on distance between the screens more than $0,5 \cdot 10^{-2} \mathrm{~m}$. and this effect is comparable with the error of temperature measurement.

\section{Conclusion}

The study and experimental data on the dependence of the temperature of the irradiated sample distance $\mathrm{x}$ to the source of thermal radiation, in the range from $8 \cdot 10^{-2} \mathrm{~m}$. to $20 \cdot 10^{-2} \mathrm{~m}$. with a source temperature of thermal radiation equal $1233,15 \mathrm{~K}$.

\section{References}

[1] Valendik E.N., CBS IV Thermal radiation from forest fires and the potential impact on the forest // boreal Coniferous zone. 2008. So 25. No. 1-2. S. 88-92

[2] URL: http://forum.vashdom.ru/threads/otopit-zagorodnyj-dom.35246/page-6 I (date of access: 18.04.2014)

[3] Kravchenko HP, Ruvinskaya OG Methods of processing the results of measurements and evaluation of errors in the teaching laboratory: a manual. Tomsk: Publishing house of Tomsk polytechnical University, 2011. $88 \mathrm{C}$

[4] Kakunin VA Statistical processing of data with a small number of experiments // Ukrainian biochemical journal. 1975. So 47. No. 6. C. 776-790 